\title{
NEUTRINO FLAVOR RATIOS ON THE EARTH FOR DECAY AND OSCILLATION SCENARIOS
}

\author{
T. C. LIU \\ Leung Center for Cosmology and Particle Astrophysics, \\ National Taiwan University, Taipei 106, Taiwan \\ tcliu@ntu.edu.tw \\ KWANG-CHANG LAI \\ Physics Group, Center for General Education, \\ Chang Gung University, Kwei-Shan 333, Taiwan \\ kcl@mail.cgu.edu.tw \\ GUEY-LIN LIN \\ Institute of Physics, National Chiao-Tung University, Hsinchu 300, Taiwan \\ glin@cc.nctu.edu.tw
}

Received 10 September 2012

Accepted 22 October 2012

Published 15 January 2013

\begin{abstract}
Due to flavor transition mechanisms in neutrino propagations, both neutrino oscillation and decay, neutrinos flavor ratio at astrophysical sources can quite different from observed ratios on the Earth. ${ }^{1}$ We use the recent global fitting result of the mixing angle values $^{2}$ to determine the allowed neutrino flavor ratios on the Earth for standard neutrino oscillation and general decay mechanisms. In this analysis, four types of the neutrino decay mechanisms can be distinguished from the oscillation case easily with arbitrary initial neutrino flavor ratio and the other four types of neutrino decay mechanisms can be distinguished from the oscillation case with the specified source. The rest types of neutrino decays strongly overlap with the oscillation and cannot be identified.
\end{abstract}

Keywords: Neutrino; mixing angle.

\section{Introduction}

The flavor ratio of astrophysical neutrinos observed on the Earth depend on both the initial flavor ratio at the source and flavor transition mechanisms during the propagation of these neutrinos. We consider not only the initial source types but also the flavor transition mechanisms with the currently measured neutrino mixing angles to analyze the allowed flavor ratios on the Earth. For the initial neutrino source part, cosmic neutrinos can be produced from various astrophysical 
objects. At production sites, different production process or environment could produce different neutrino flavor ratios. ${ }^{3}$ Typical hadronic interaction produces neutrinos through $\pi^{ \pm} \rightarrow \mu^{ \pm} \rightarrow e^{ \pm}$chains and generates the neutrino flavor ratio, $\left\{\phi\left(\nu_{e}\right), \phi\left(\nu_{\mu}\right), \phi\left(\nu_{\tau}\right)\right\}=\{1,2,0\}$, which is referred to as pion source. When the sources are hidden behind strong field or dense matters, muon may lose energy before decay, so that the muon neutrinos and electron neutrinos from muon decays could become very low energy and consequently undetectable by astrophysical neutrino telescopes. This source, is referred to as muon-damped source with the flavor ratio $\left\{\phi\left(\nu_{e}\right), \phi\left(\nu_{\mu}\right), \phi\left(\nu_{\tau}\right)\right\}=\{0,1,0\} .{ }^{4-6}$ However, because of neutrino oscillation, their flavor ratio changes while neutrinos are on-route to the Earth and being detected. Since the effect of muon energy loss increases with energy, therefore the smooth transition of the flavor ratios from pion source to muon-damped as the neutrino energy increases is expected. ${ }^{6,7}$ We introduce this smooth transition as more general source, $\left\{\phi_{0}\left(\nu_{e}\right): \phi_{0}\left(\nu_{\mu}\right): \phi_{0}\left(\nu_{\tau}\right)\right\}=\{\alpha: 1-\alpha: 0\}$, with $0<\alpha \leq 1 / 3$. Concerning the prorogation part, standard three-flavors neutrino oscillation and arbitrary neutrino decay mechanisms are considered. We ignore the different between the particle and antiparticle in the decay mechanisms to simplify the calculation.

\section{Standard Oscillation Mechanism}

In neutrino oscillation theory, the relationship between neutrino mass eigenstates and neutrino flavor eigenstates are described by Pontecorvo-Maki-Nakagawa-Sakata mixing matrix $U, 8,9$ which is formed by three mixing angles $\theta_{i j}$ and the $C P$ violation phase $\delta$.In the limit of large neutrino propagation distance, the neutrino oscillation probability only depend on the mixing angles $\theta_{i j}$ and $C P$ phase. In this case, the transition probability for neutrino flavor $\beta$ to the other flavor $\alpha$ can be expressed in terms of unitary matrix $U$ purely:

$$
P_{\alpha \beta}=\sum_{i=1}^{3}\left|U_{\alpha i}\right|^{2}\left|U_{\beta i}\right|^{2} .
$$

Hence he neutrino flux at the astrophysical site and that detected on the Earth is related by

$$
\left(\begin{array}{c}
\phi\left(\nu_{e}\right) \\
\phi\left(\nu_{\mu}\right) \\
\phi\left(\nu_{\tau}\right)
\end{array}\right)=\left(\begin{array}{lll}
P_{e e} & P_{e \mu} & P_{e \tau} \\
P_{\mu e} & P_{\mu \mu} & P_{\mu \tau} \\
P_{\tau e} & P_{\tau \mu} & P_{\tau \tau}
\end{array}\right)\left(\begin{array}{l}
\phi_{0}\left(\nu_{e}\right) \\
\phi_{0}\left(\nu_{\mu}\right) \\
\phi_{0}\left(\nu_{\tau}\right)
\end{array}\right) \equiv P\left(\begin{array}{c}
\phi_{0}\left(\nu_{e}\right) \\
\phi_{0}\left(\nu_{\mu}\right) \\
\phi_{0}\left(\nu_{\tau}\right)
\end{array}\right)
$$

where $\phi\left(\nu_{\alpha}\right)$ is the neutrino flux measured on the Earth while $\phi_{0}\left(\nu_{\alpha}\right)$ is the neutrino flux at the source, and the matrix element $P_{\alpha \beta}$ is the probability for the oscillation $\nu_{\beta} \rightarrow \nu_{\alpha}$. New results on neutrino mixing angles, listed in Table 1, are introduced into Equation (2). The allowed neutrino flavor ratios on the Earth from the general neutrino sources, $\left\{\phi_{0}\left(\nu_{e}\right): \phi_{0}\left(\nu_{\mu}\right): \phi_{0}\left(\nu_{\tau}\right)\right\}=\{\alpha: \beta: 1-\alpha-\beta\}$, with $\alpha, \beta>0$ and $\alpha+\beta \leq 1$, via oscillation mechanism are shown in Fig. 2. The uncertainties of neutrino mixing angles of both hierarchies are similar at the $1 \sigma \mathrm{CL}$, hence the 
Table 1. Neutrino mixing angles.

\begin{tabular}{clcc}
\hline Parameters & Best fit & $1 \sigma$ & $3 \sigma$ \\
\hline $\sin ^{2} \theta_{12}$ & 0.307 & $0.291-0.325$ & $0.259-0.359$ \\
$\sin ^{2} \theta_{13}$ (Normal hierarchy) & 0.0245 & $0.214-0.279$ & $0.149-0.344$ \\
$\sin ^{2} \theta_{13}$ (Inverted hierarchy) & 0.0246 & $0.0215-0.0280$ & $0.150-0.347$ \\
$\sin ^{2} \theta_{23}$ (Normal hierarchy) & 0.398 & $0.372-0.428$ & $0.330-0.638$ \\
$\sin ^{2} \theta_{23}$ (Inverted hierarchy) & 0.408 & $0.378-0.443$ & $0.335-0.658$ \\
\hline
\end{tabular}

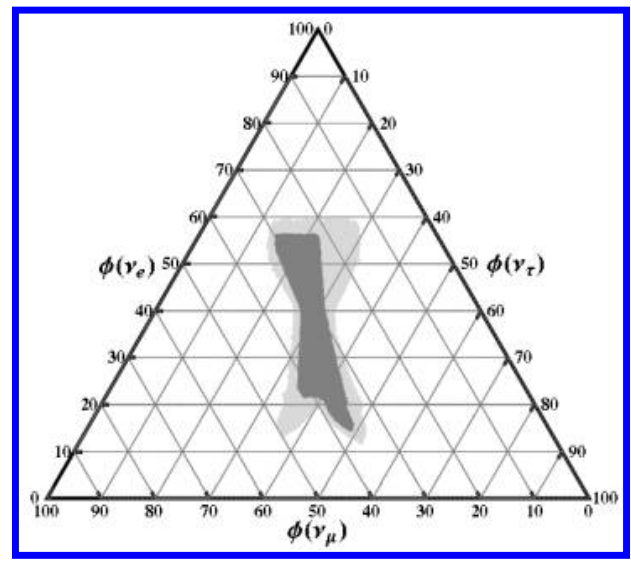

(a) Normal mass hierarchy

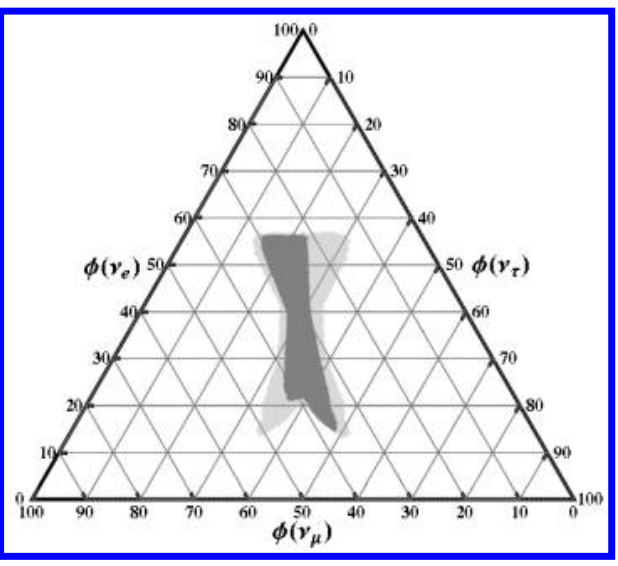

(b) Inverted mass hierarchy

Fig. 1. The ranges for the neutrino flavor ratios on the Earth resulting from standard neutrino oscillation for normal and inverted mass hierarchy. Both panels show the range of neutrino flavor ratios from the source flavor ratios, $\left\{\phi_{0}\left(\nu_{e}\right): \phi_{0}\left(\nu_{\mu}\right): \phi_{0}\left(\nu_{\tau}\right)\right\}=\{\alpha: \beta: 1-\alpha-\beta\}$, with $\alpha>0$, $\beta>0$ and $\alpha+\beta \leq 1$. The grey and light grey colors correspond to the $1 \sigma$ and $3 \sigma$ CL of neutrino mixing angles, respectively. The values of mixing angles are taken as the Table 1.

allowed flavors ratios on the Earth are identical. We also consider the standard sources, pion and muon-damped sources, and the uncertainties of mixing angles. The allowed flavor ratios are shown in Fig. 1. The allowed neutrino flavor ratios on the Earth for the pion source and muon-damped source are centered at $\{1: 1: 1\}$ and $\{5: 8: 7\}$.

\section{Neutrino Decay Mechanism}

In neutrino decay mechanisms, we consider only two-body decay modes with three active neutrino flavors and assume the decays are complete. As discussed in Ref. 10, the probability transition matrix characterizing the decay scenario is written in terms of PMNS matrix and corresponding branching ratios

$$
P_{\alpha \beta}^{d e c}=\sum_{i \text { stable }}\left(\left|U_{\alpha i}\right|^{2}+\sum_{j \text { unstable }}\left|U_{\alpha j}\right|^{2} \mathrm{Br}_{j \rightarrow i}\right)\left|U_{\beta i}\right|^{2},
$$




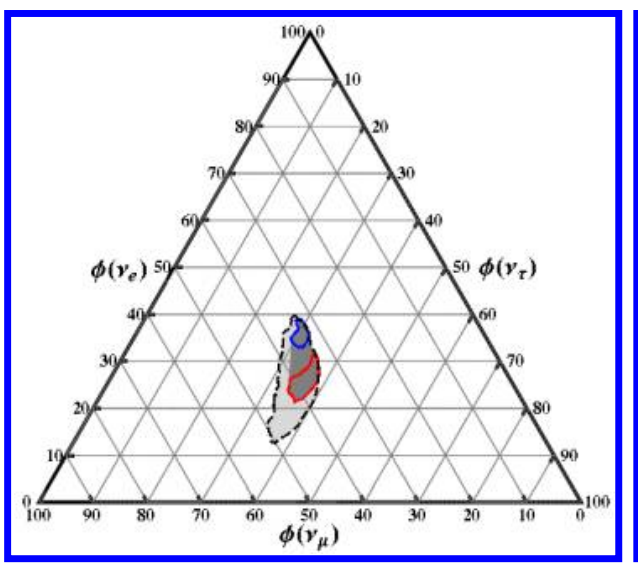

(a) Normal mass hierarchy

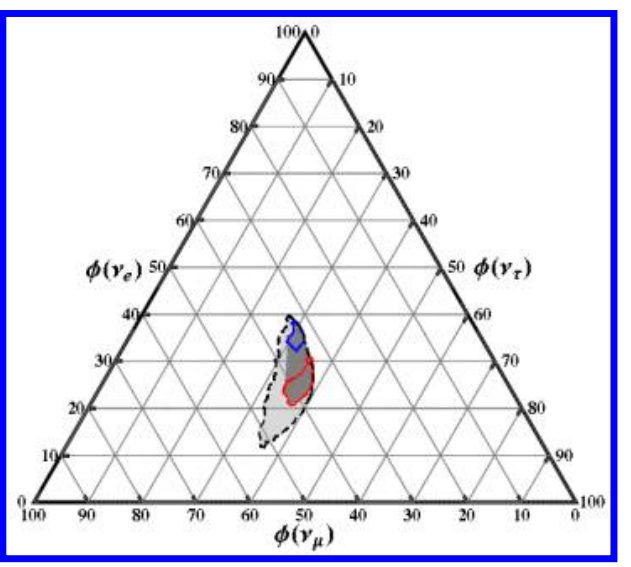

(b) Inverted mass hierarchy

Fig. 2. (color online) The ranges for the neutrino flavor ratios on the Earth resulting from standard neutrino oscillation for normal and inverted mass hierarchy. Both panels show the range of neutrino flavor ratios from the source flavor ratios, $\left\{\phi_{0}\left(\nu_{e}\right): \phi_{0}\left(\nu_{\mu}\right): \phi_{0}\left(\nu_{\tau}\right)=\{\alpha: 1-\alpha: 0\}\right.$, with $0 \leq \alpha \leq 1$. The grey and light grey region with dashed line correspond to the $1 \sigma$ and $3 \sigma$ CL of neutrino mixing angles, respectively. Blue and red curves correspond to the allowed neutrino flavor ratios from the pion source and muon-damped source at $1 \sigma$ CL of neutrino mixing angles. The values of mixing angles are the same as in Table 1.

Table 2. The neutrino decay and oscillation scenarios. The suffix "H", "M", and "L" label the heaviest, middle and lightest mass eigenstates. The number " 1 ", "2" and " 3 " label $\nu_{1}, \nu_{2}$ and $\nu_{3}$ mass eigenstates. The numbers in italic and those in black correspond to the unstable and stable eigenstates, respectively.

\begin{tabular}{c|c|ccc|cc}
\hline Decay type & Hierarchy & H & M & L & Scenarios & Figure label \\
\hline & & & & Oscillation & Fig. 2 & Fig. 3a \\
N1 & Normal & 3 & 2 & 1 & Heaviest \& middle decay & Fig. 3c \\
\hline N1 & Inverted & 2 & 1 & 3 & Heaviest \& middle decay & Fig. 4a \\
I2 & Normal & 3 & 2 & 1 & Heaviest decay & Fig. 5c \\
\hline N3 & Inverted & 2 & 1 & 3 & Middle decay & Fig. 5a \\
I3 & Inverted & 2 & 1 & 3 & Middle decay & Fig. 4c \\
\hline N4 & Normal & 3 & 2 & 1 & Lightest decay to invisible & Fig. 4b \\
I4 & Inverted & 2 & 1 & 3 & Lightest decay to invisible & Fig. 4d \\
\hline N5 & Normal & 3 & 2 & 1 & Middle decay \& Lightest & Fig. 3b \\
I5 & Inverted & 2 & 1 & 3 & decay to invisible & Fig. 5d \\
\hline N6 & Normal & 3 & 2 & 1 & Heaviest decay \& Lightest & Fig. 5b \\
I6 & Inverted & 2 & 1 & 3 & decay to invisible & Fig. 3d \\
\hline
\end{tabular}


where indices $i$ and $j$ denote mass eigenstates and $\mathrm{Br}_{i \rightarrow j}$ shows branching ratio for unstable state $i$ decays to stable state $j . \sum_{f} \mathrm{Br}_{i \rightarrow j}=1$ for $i$ state fully decays to $j$ state. In general, $\sum_{f} \mathrm{Br}_{i \rightarrow j}<1$ and $\mathrm{Br}_{i \rightarrow \text { invisible }}=1-\sum_{f} \mathrm{Br}_{i \rightarrow j}$ denotes the probability for $i$ state decays to invisible states. The invisible states are invisible for neutrino detectors, those invisible states is sterile neutrino. Table 2 lists all possibilities of decays with oscillation included for comparison. We note that a detailed statistical analysis has been performed in Ref. 10 for distinguishing various decay scenarios from the standard neutrino oscillation. The analysis there is based on future measurements of track to shower event ratio in neutrino telescopes. We shall however consider more types of event ratio for distinguishing different flavor transition mechanisms. In stead of performing detailed statistical analysis, we shall focus on some interesting qualitative features.

\subsection{Fully distinguishable case: The allowed flavor ratios on the Earth for decay scenarios can rule out from neutrino oscillation one}

In Fig. 3, The scenarios "N1", "N5", "I1", and "I6" can be fully separated from the standard neutrino oscillation scenarios for both pion and muon-damped sources at $3 \sigma$ CL of neutrino mixing angles. The allowed electron neutrino flavor fractions of "N5" and "I1" scenarios are approaching 0.

\subsection{Partially distinguishable case: The allowed flavor ratios on the Earth for decay scenarios can be distinguished from neutrino oscillation one}

In Fig. 4, the allowed neutrino flavor ratios on the Earth from muon-damped source by oscillations can be distinguished from decay scenarios "N2" and "I4" at $3 \sigma$ CL. However, the allowed neutrino flavor ratios on the Earth from pion source by os-

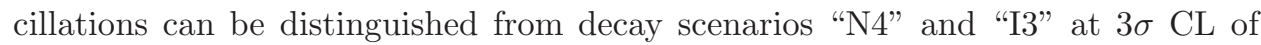
neutrino mixing angles.

\subsection{Overlapped case: The allowed flavor ratios on the Earth for decay scenarios and those under oscillations cannot be distinguished}

In Fig. 5, the allowed neutrino flavor ratios on Earth by decay scenarios "N3", "N6", "I2", and "I5" strongly overlap those by standard neutrino oscillation for both sources.

\subsection{Conclusion}

From experimental point of view, electron neutrino event and tau neutrino event are not easy to separate. Muon neutrino events can be easily isolated from the others 


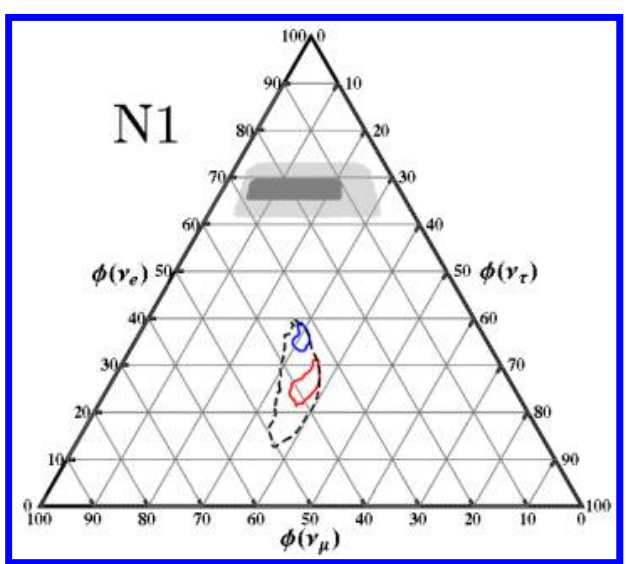

(a) The heaviest and middle mass eigenstates are unstable and decay into the lightest and invisible eigenstates for normal mass hierarchy. In this case, the decay scenario is fully distinguishable from the standard oscillation mechanism in $3 \sigma$ CL of neutrino mixing angles.

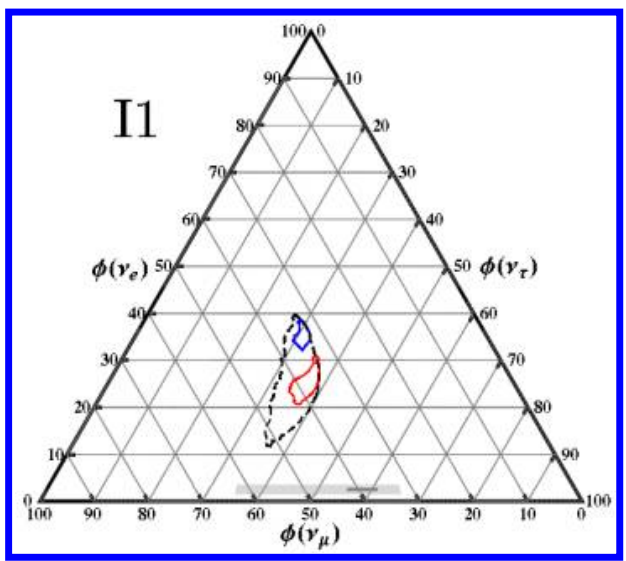

(c) The heaviest and middle mass eigenstates are unstable and decay into the lightest and invisible eigenstates for inverted mass hierarchy. In this case, the decay scenario is fully distinguishable from the standard oscillation mechanism in $3 \sigma$ CL of neutrino mixing angles.

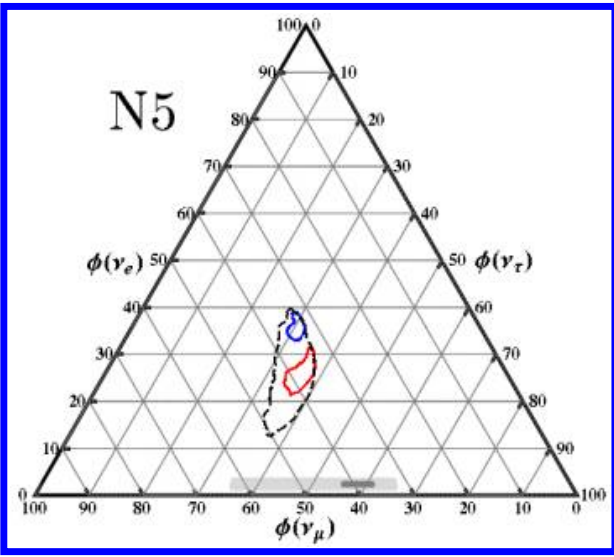

(b) The middle and lightest mass eigenstates are unstable and decay into the invisible eigenstates. In this case, the decay scenario is fully distinguishable from the standard oscillation mechanism in $3 \sigma$ CL of neutrino mixing angles.

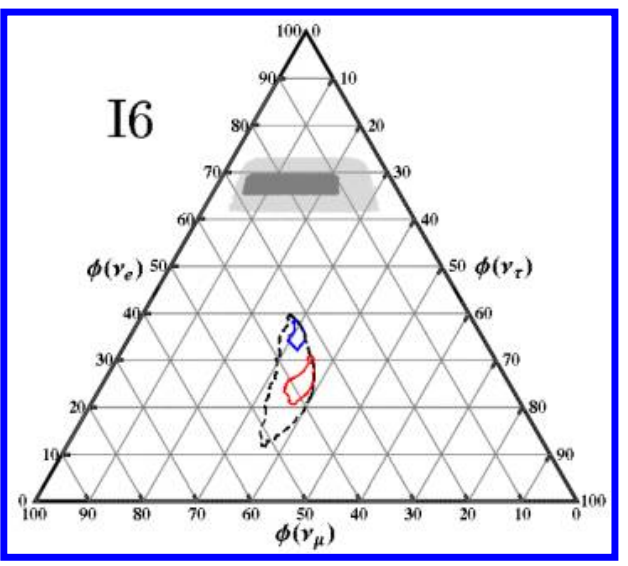

(d) The heaviest and lightest mass eigenstates are unstable and decay into invisible eigenstate for inverted mass hierarchy. In this case, the decay scenario is fully distinguishable from the standard oscillation mechanism in $3 \sigma \mathrm{CL}$ of neutrino mixing angles.

Fig. 3. (color online) The grey and light grey regions denote allowed neutrino flavor ratios on the Earth at $1 \sigma$ and $3 \sigma$ CL of neutrino mixing angles for initial flavor ratio, $\left\{\phi_{0}\left(\nu_{e}\right): \phi_{0}\left(\nu_{\mu}\right)\right.$ : $\left.\phi_{0}\left(\nu_{\tau}\right)\right\}=\{\alpha: 1-\alpha: 0\}$, with $0 \leq \alpha \leq 1$. Regions enclosed by blue and red curves as well as the region enclosed by the dashed curve correspond to standard oscillation case with different initial flavor ratios as described in Fig. 2. 


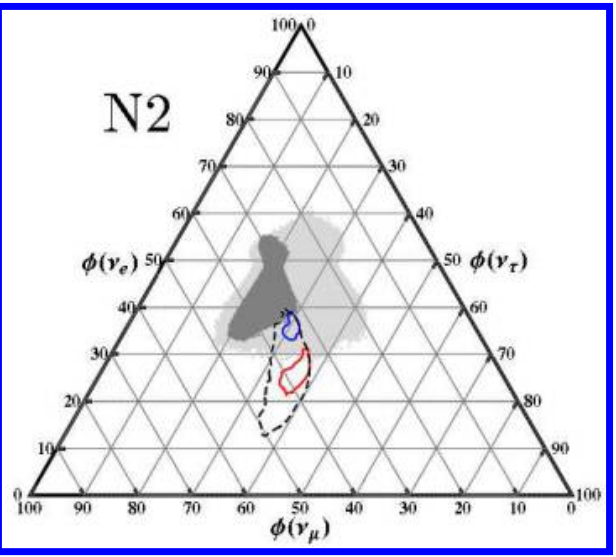

(a) The unstable heaviest mass eigenstate decays into the lighter mass eigenstates for normal mass hierarchy. The allowed flavor ratios on the Earth from the muon-damped source can be separated from the decay scenario at $1 \sigma$ CL of neutrino mixing angles.

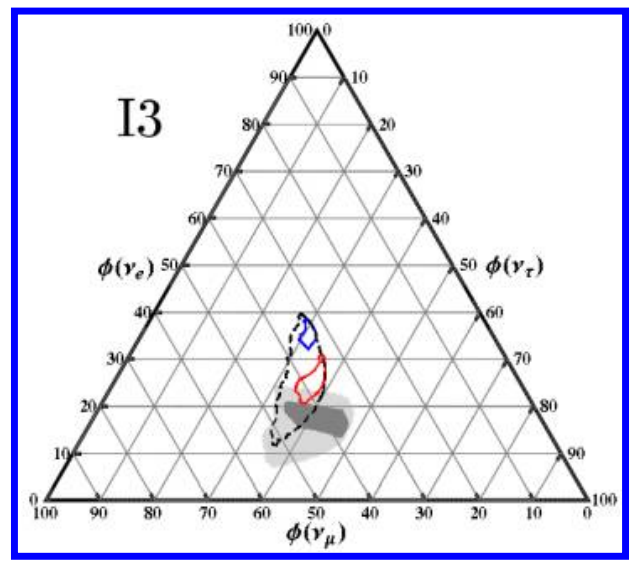

(c) The unstable middle mass eigenstate decays into the lighter mass eigenstates for inverted mass hierarchy. The allowed flavor ratios on the Earth from the pion source can be separated from the decay scenario at $3 \sigma$ CL of neutrino mixing angles.

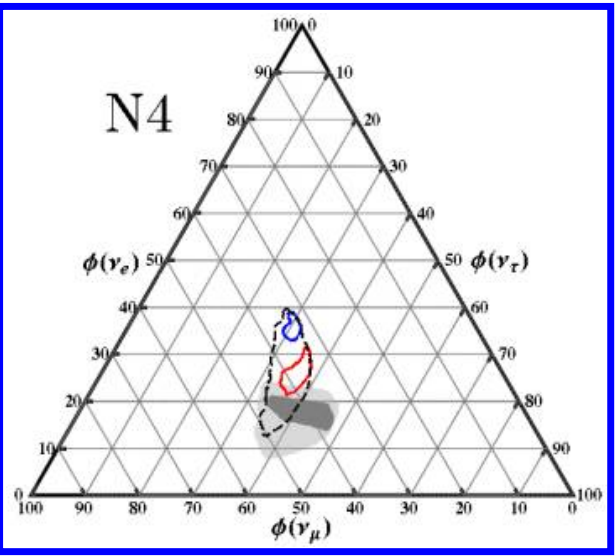

(b) The unstable lightest mass eigenstate decays into the invisible mass eigenstate for normal mass hierarchy only. The allowed flavor ratios on the Earth from the pion source can be separated from the decay scenario at $3 \sigma$ CL of neutrino mixing angles.

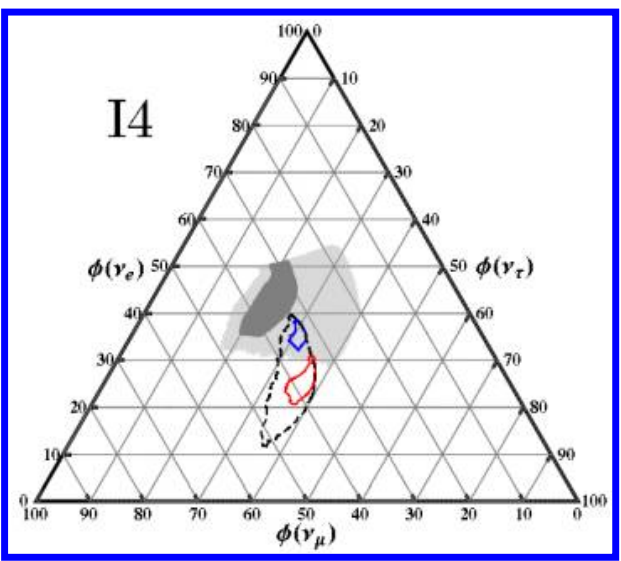

(d) The unstable lightest mass eigenstate decays into the invisible mass eigenstate for inverted mass hierarchy only. The allowed flavor ratios on the Earth from the muon-damped source can be separated from the decay scenario at $1 \sigma \mathrm{CL}$ of neutrino mixing angles.

Fig. 4. (color online) The grey and light grey regions denote allowed neutrino flavor ratios on the Earth at $1 \sigma$ and $3 \sigma$ CL of neutrino mixing angles for initial flavor ratio, $\left\{\phi_{0}\left(\nu_{e}\right): \phi_{0}\left(\nu_{\mu}\right)\right.$ : $\left.\phi_{0}\left(\nu_{\tau}\right)\right\}=\{\alpha: 1-\alpha: 0\}$, with $0 \leq \alpha \leq 1$. Regions enclosed by blue and red curves as well as the region enclosed by the dashed curve correspond to standard oscillation case with different initial flavor ratios as described in Fig. 2. 


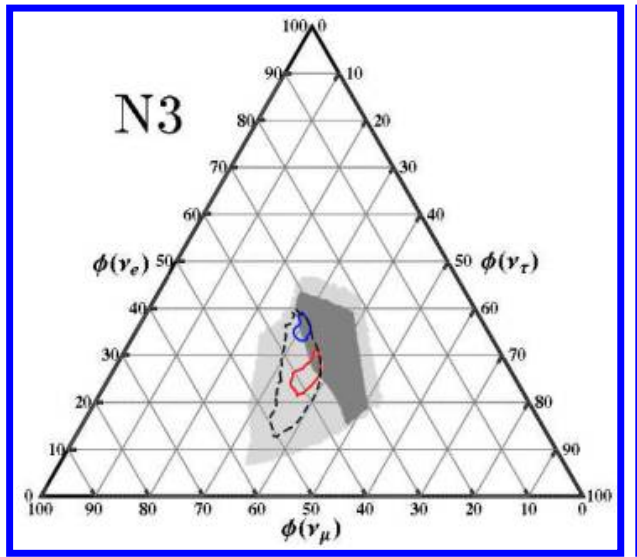

(a) The unstable middle mass eigenstate decays into the lighter mass eigenstates for normal mass hierarchy. The allowed flavor ratios on the Earth from both muon damped source and pion source cannot be separated from the decay scenario in $3 \sigma \mathrm{CL}$ of neutrino mixing angles.

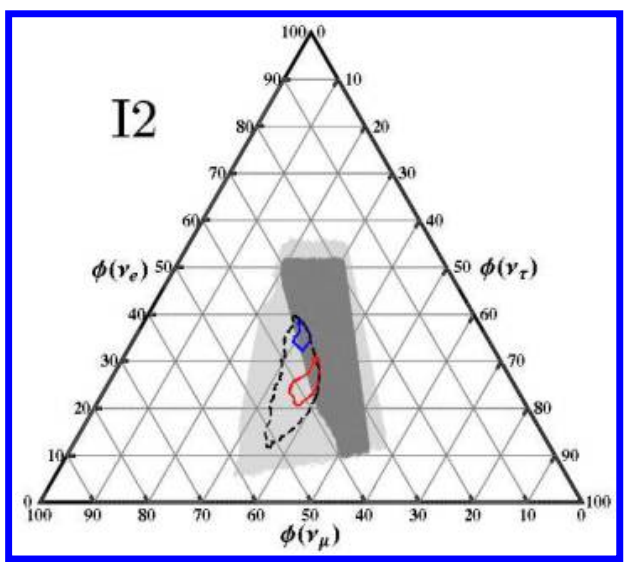

(c) The unstable heaviest mass eigenstate decays into the lighter mass eigenstates for inverted mass hierarchy. The allowed flavor ratios on the Earth from both muon damped source and pion source cannot be separated from the decay scenario.

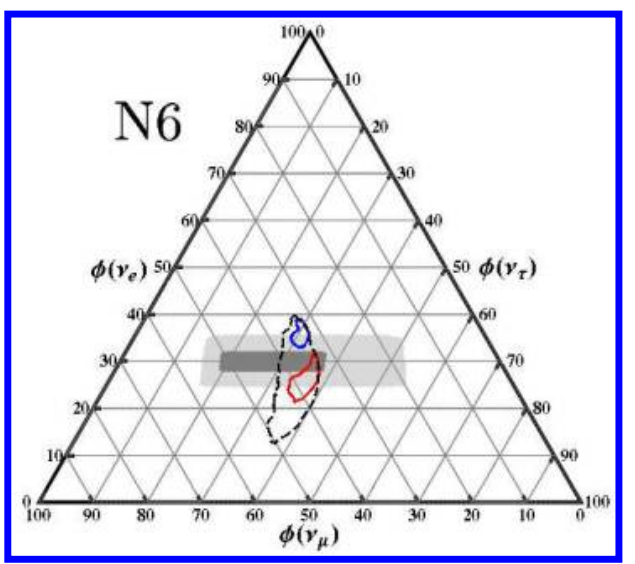

(b) The heaviest and lightest mass eigenstates are unstable and decay into invisible eigenstate for normal mass hierarchy. The allowed flavor ratios on the Earth from both muon damped source and pion source cannot be separated from the decay scenario in $3 \sigma$ CL of neutrino mixing angles.

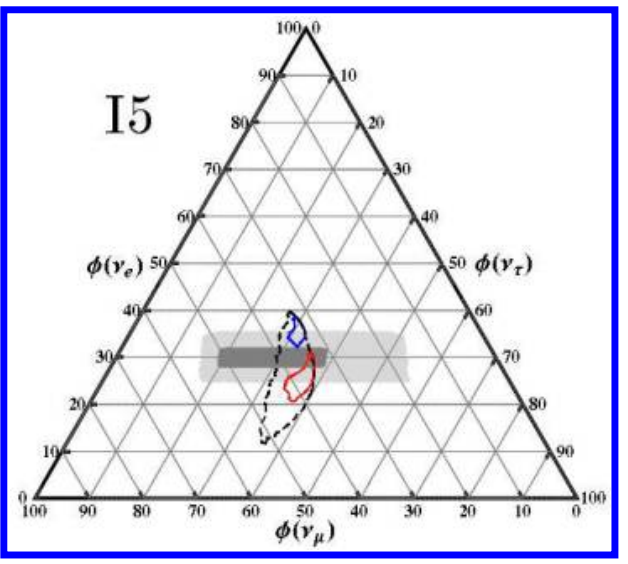

(d) The middle and lightest mass eigenstates are unstable and decay into the invisible eigenstate for inverted mass hierarchy. The allowed flavor ratios on the Earth from both muon damped source and pion source cannot be separated from the decay scenario.

Fig. 5. (color online) The grey and light grey regions denote allowed neutrino flavor ratios on the Earth at $1 \sigma$ and $3 \sigma \mathrm{CL}$ of neutrino mixing angles for initial flavor ratio, $\left\{\phi_{0}\left(\nu_{e}\right): \phi_{0}\left(\nu_{\mu}\right)\right.$ : $\left.\phi_{0}\left(\nu_{\tau}\right)\right\}=\{\alpha: 1-\alpha: 0\}$, with $0 \leq \alpha \leq 1$. Regions enclosed by blue and red curves as well as the region enclosed by the dashed curve correspond to standard oscillation case with different initial flavor ratios as described in Fig. 2. 
by its track-like behavior. Hence "N5" and "I1" decay scenarios are not distinguishable from the standard oscillation scenario. On the other hand, "N1" and "I6" can be distinguished from the standard oscillation scenario. In the extremely high energy neutrino case, the behavior of tau neutrino event is becoming similar to the behavior of muon neutrino events. ${ }^{16}$ Electron neutrino event is the one that can be separated from the other two types of events. In this case, the decay scenarios "N1", "N5", "I1", and "I6" can be easily ruled out or confirmed. However, due to the degeneracy, we cannot further distinguish "N1" and "I6" scenarios or "N5" and "I1" scenarios. We have also found that "N4" and "I3" decay scenarios can be well separated from the standard oscillation scenario in the case of pion source, but not in the case of muon-damped source. On the other hand, "N2" and "I4" decay scenarios can be well separated from the standard oscillation scenario in the case of muon-damped source, but not in the case of pion source.

\section{Acknowledgments}

G.L.L. is supported by National Science Council of Taiwan under Grant No. 992112-M-009-005-MY3. K. C. Lai is supported by National Science Council of NSC100-2110-M-182-001-MY3.

\section{References}

1. M. Maltoni and W. Winter, arXiv:0803.2050v2 [hep-ph].

2. G. L. Fogli, E. Lisi, A. Marrone, D. Montanino, A. Palazzo and A. M. Rotunno, arXiv:1205.5254 [astro-ph].

-3. S. Pakvasa, Mod. Phys. Lett. A 19, 1163 (2004); Yad. Fiz. 67, 1179 (2004).

4. M. Kachelriess, S. Ostapchenko and R. Tomas, Phys. Rev. D 77, 023007 (2008).

5. J. P. Rachen and P. Meszaros, Phys. Rev. D 58, 123005 (1998).

6. T. Kashti and E. Waxman, Phys. Rev. Lett. 59, 181101 (2005).

7. M. Kachelriess and R. Tomas, Phys. Rev. D 74, 063009 (2006), arXiv:0606406 [astro$\mathrm{ph}$.

8. Z. Maki, M. Nakagawa and S. Sakata, Prog. Theor. Phys. 28, 870 (1962).

9. B. Pontecorvo, Zh. Eksp. Teor. Fiz. 53, 1717 (1967).

10. M. Maltoni and W. Winter, JHEP 64, 0807 (2008).

11. R. L. Awasthi and S. Choubey, Phys. Rev. D 76, 113002 (2007), arXiv:0706.0399 [hep-ph].

12. S.-L. Chen, X.-G. He and H.-C. Tsai, arXiv:0707.0187 [hep-ph].

13. S. Zhou, arXiv:0706.0302 [hep-ph].

14. X.-Q. Li, Y. Liu and Z.-T. Wei, arXiv:0707.2285 [hep-ph].

15. D. Majumdar, arXiv:0708.3485 [hep-ph].

16. T. C. Liu, M. A. Huang and G.-L. Lin, arXiv:1004.5154 [hep-ph]. 